\title{
Development of substorm bulges during different solar wind structures
}

\author{
I. V. Despirak ${ }^{1}$, A. A. Lubchich ${ }^{1}$, A. G. Yahnin ${ }^{1}$, B. V. Kozelov ${ }^{1}$, and H. K. Biernat ${ }^{2}$ \\ ${ }^{1}$ Polar Geophysical Institute, Apatity, Russia \\ ${ }^{2}$ Space Research Institute, Graz, Austria
}

Received: 27 August 2008 - Revised: 23 March 2009 - Accepted: 13 April 2009 - Published: 4 May 2009

\begin{abstract}
Using data from WIND spacecraft, we investigated the difference in substorm bulge development during different types of solar wind flow: solar wind recurrent streams (RS), corotating interaction regions (CIR), magnetic clouds (MC), and the region of interaction of magnetic clouds with undisturbed solar wind (Sheath). The RS/CIR and MC/Sheath structures were examined for the periods December 1996-July 1997; January 2000-December 2000; October 2001. All available auroral substorms observed by the Ultra Violet Imager onboard the Polar spacecraft during these periods were studied. It is shown that the largest latitudinal and longitudinal sizes of the auroral bulge expansions are during CIR and Sheath intervals. We found a difference in auroral bulge parameters for MC- and RS-associated substorms. In contrast to substorms associated with RS, the latitudinal size of the auroral bulge during MC is smaller, but longitudinal size is larger. As consequence, the ratio between longitudinal and latitudinal sizes for MC-associated substorms is also larger. We suggest that the latter feature is explained by different configuration of the near-Earth magnetotail during RS and MC.
\end{abstract}

Keywords. Magnetospheric physics (Auroral phenomena; Solar wind-magnetosphere interactions; Storms and substorms)

\section{Introduction}

It is variations in the solar wind conditions that majorly govern the development of substorm disturbances. The substorm poleward expansion, as well as auroral bulge formation and dynamics, are determined by the amount of energy accumulated in the magnetospheric lobes, which, in turn, depends on

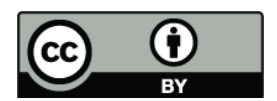

Correspondence to: I. V. Despirak (despirak@pgia.ru) the solar wind parameters (Shukhtina et al., 2005; Miyashita et al., 2003). The progress of substorm activity down the magnetospheric tail, which is displayed in the ionosphere as a substorm poleward expansion up to high geomagnetic latitudes, is controlled by the solar wind velocity $V_{X}$, and the southward component $B_{Z}$ of the interplanetary magnetic field (Gussenhoven, 1982; Zverev et al., 1979; Dmitrieva and Sergeev, 1984; Yahnin et al., 2004). At the same time, it has been shown that at high latitudes, substorms occur more frequently during solar minimum conditions (Dmitrieva and Sergeev, 1984; Despirak et al., 2008), and correlate with the solar wind recurrent streams (Sergeev et al., 1979). This means that substorm expansion is not governed solely by $V_{X}$ and $B_{Z}$ IMF but also by the structure of solar wind flow.

Solar wind flow may be different depending on the state of solar activity. Thus during solar minimum, the recurrent streams (RS) originating from coronal magnetic holes, characterized by 27-day recurrence, are predominant (Krieger et al., 1973; Pudovkin, 1996). During solar maximum, most common are sporadic flows associated with coronal mass ejections (CME) (Wang and Sheeley, 1994; Webb and Howard, 1994). Near the Earth they are observed as magnetic clouds (MC) (e.g., Burlaga et al., 1982). Ahead of both $\mathrm{MC}$ and RS, the regions of interaction with undisturbed solar wind (Sheath and CIR) are known to form, which are characterized by high density, increased pressure and strong IMF variability.

To relate peculiarities in substorm dynamics to varying solar wind conditions, it is important to understand how different solar wind structures affect the substorm expansion. In this study, we consider substorm development against a background of magnetic clouds (MC), recurrent streams (RS), and regions of their interaction with undisturbed solar wind (Sheath and CIR). It should be noted that three of these structures, namely, Sheath, CIR, and MC, are the sources of geomagnetic storms (e.g., Gonzalez et al., 1990; Tsurutani et al., 2006). Furthermore, the storms associated with MC,

Published by Copernicus Publications on behalf of the European Geosciences Union. 
Sheath, and CIR, differ in intensity, recovery phase duration, etc. (e.g., Huttunen et al., 2006; Pulkkinen et al., 2007; Yermolaev and Yermolaev, 2006). Therefore, we will search for distinctions in the development of substorms occurring during both geomagnetic storms connected with the MC, Sheath and CIR, and rather quiet "non-storm" periods of RS.

The purpose of this study is to clear up how four different solar wind structures (MC, Sheath, CIR, and RS) affect the auroral bulge development. We use the optical data of substorm development from the Polar satellite which are compared with interplanetary medium parameters measured by the Wind satellite.

\section{Data}

The auroral bulge development is studied by Polar UVI data in the LBHL band (1600-1800 $\AA$ ). A special procedure is used for determination of the boundary and the size of the auroral bulge from the Polar UVI images. The luminosity of the UV aurora is divided into 25 intervals according to $I=I_{0} \cdot 10^{\Delta \cdot n}$, where $I_{0} \sim 3.2$ photons $\mathrm{cm}^{-2} \mathrm{~s}^{-1}, \Delta=0.1$, and $n$ is the interval number. A background level of the auroral oval luminosity for every considered substorm was determined as the luminosity observed before the substorm onset at the onset meridian. The auroral bulge area is defined as the area where the auroral luminosity is at the level next above the background. Typically, under relatively quiet (non-storm) conditions, this corresponds to level of the photon flux of $10-25$ photons $\mathrm{cm}^{-2} \mathrm{~s}^{-1}$. However, under storm-time conditions the selected level for the auroral bulge determination can reach up to 200 photons $\mathrm{cm}^{-2} \mathrm{~s}^{-1}$. Further, the onset latitude $\left(L_{0}\right)$ is determined as the lower latitude at the selected luminosity level of the first UV aurora spot during the substorm. The same selected luminosity level is used to control the auroral bulge expansion (note, that for this only spots connected with the onset one was considered, not the other, separated spots at different locations). The same luminosity level is used to define the maximal latitude of the bulge development $\left(L_{m}\right)$ as well as maximal latitudinal $\left(L_{F}\right)$ and longitudinal dimensions of the bulge $\left(L_{d}\right)$.

As assumed, the storms and substorms are different magnetospheric/ionospheric dynamical processes (e.g., Tsurutani and Gonzalez, 2006). If a substorm occurs during the storm time, it has the same typical signatures as a substorm developing during non-storm conditions. Namely, on the Polar UVI auroral images one can see: (1) the burst of luminous spot, (2) expansion of spot to the pole and in longitude, and (3) decay of spot. Development of substorm bulge during the storm time has the same time scale as "usual" substorms, i.e., from $10 \mathrm{~min}$ up to an hour. Therefore we will determine the parameters of auroral bulge during storm-time conditions in the same way as for "usual" substorms, of course, taking into account the higher background level of the auroral oval luminosity during storm-time conditions.
The solar wind and interplanetary magnetic field parameters were taken from the WIND satellite data (SWE and MFI data with 1 min resolution were used). The $\mathrm{X}$-component of the solar wind velocity $V_{X}$, the southward IMF component $B_{S}$, and dawn-dusk component of the interplanetary electric field $E_{W}$ are chosen as measure of the solar wind/IMF influence. We used the values of these parameters averaged over two hours up to the time of maximum substorm development, i.e., $\bar{V}_{X}=N^{-1} \cdot \sum_{i=1}^{N} V_{X i}, \bar{B}_{S}=N^{-1} \cdot \sum_{i=1}^{N} B_{S i}\left(B_{S i}=-B_{Z i}\right.$, for $B_{Z i} \leq 0$, and $B_{S i}=0$, otherwise), and $\bar{E}_{W}=N^{-1} \cdot \sum_{i=1}^{N} E_{W i}$ $\left(E_{W i}=E_{Y i} \equiv\left[\boldsymbol{V}_{i} \times \boldsymbol{B}_{i}\right]_{Y}\right.$, for $E_{Y i} \geq 0$, and $E_{W i}=0$, otherwise), where $N \sim 120$. Two hours averaging interval was chosen to include both the growth phase $\sim 1 \mathrm{~h}$ (see, e.g., McPherron, 1970) and expansive phase $\sim 1 \mathrm{~h}$ (see, e.g., Akasofu, 1964) up to maximum substorm development, since energy input from the solar wind to the magnetosphere may continue during substorm explosive phase as well, if the interplanetary magnetic field (IMF) keeps the southward direction.

The magnetic clouds (MC) are characterized as regions, where the magnetic field strength is higher than the average, the density is relatively low, the magnetic pressure strongly exceeds the ion thermal pressure, the magnetic field direction changes through the cloud by rotating parallel to a plane which is highly inclined with respect to the ecliptic (Burlaga et al., 1982).

In front of the magnetic cloud there is a region of interaction with undisturbed solar wind (Sheath). This region is characterized by an increase in pressure, temperature, and density of the solar wind.

Two examples of magnetic cloud observation are shown in Fig. 1. On the left panels, one can see the SW and IMF data during the passage of second magnetic cloud of the Bastille Day event (15-16 July 2000). Here we have used the Geotail satellite data from OMNI set because of a temporary gap in the WIND data during this event. On the right panels, the WIND data during MC on 17-18 September 2000 are given.

The events of magnetic clouds as well as the start and finish times of their observation were taken from: http: //lepmfi.gsfc.nasa.gov/mfi/mag_cloud_pub1.html. All auroral substorms observed by Polar during $15 \mathrm{MCs}$ for the year 2000, in October 2001, December 1996 and from January to July 1997 were studied. All substorms were divided into 2 groups: substorms observed during the passage of the magnetic clouds ( 21 events, 1 st group), of the Sheath (11 events, 2nd group). For 6 magnetic clouds, substorms have been observed from Polar during both Sheath and MC, whereas for 5 (4) events substorms have been observed only during the passage of one structure: MC (Sheath). In 3 cases from 21 events of 1st group the substorms occurred in the beginning of the magnetic cloud when, e.g., a substorm growth phase was realized during the Sheath interval and an expansive phase occurred at the time of MC passage. In the other 


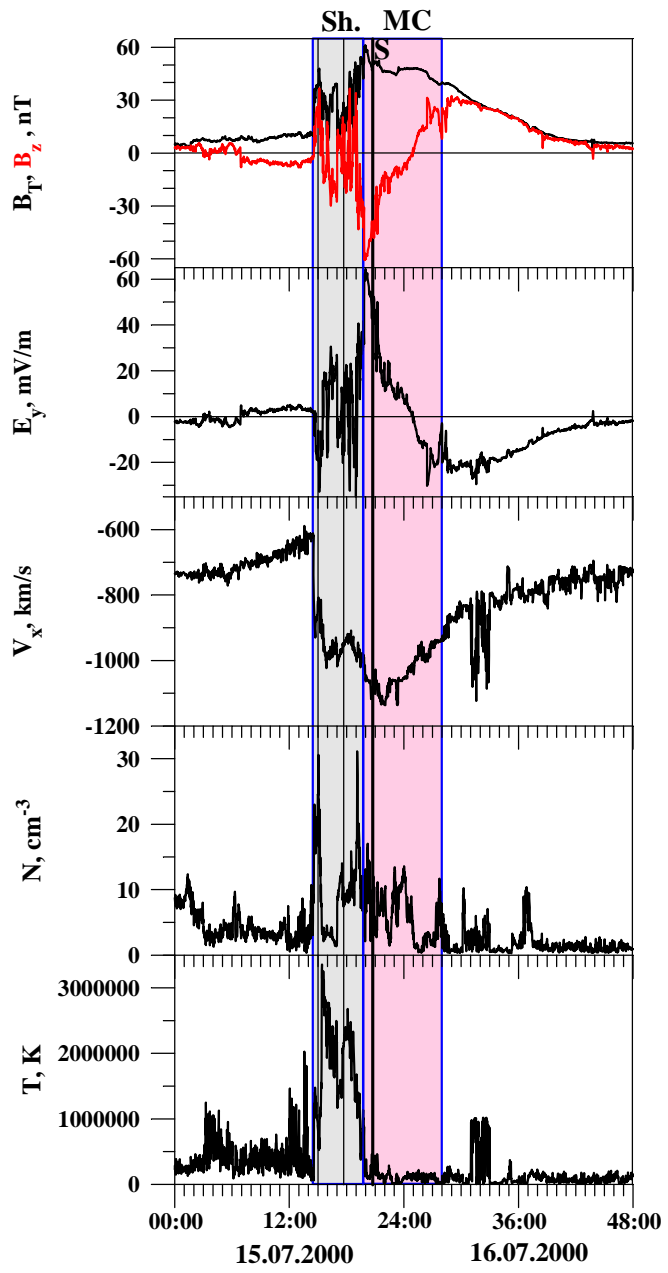

(a)

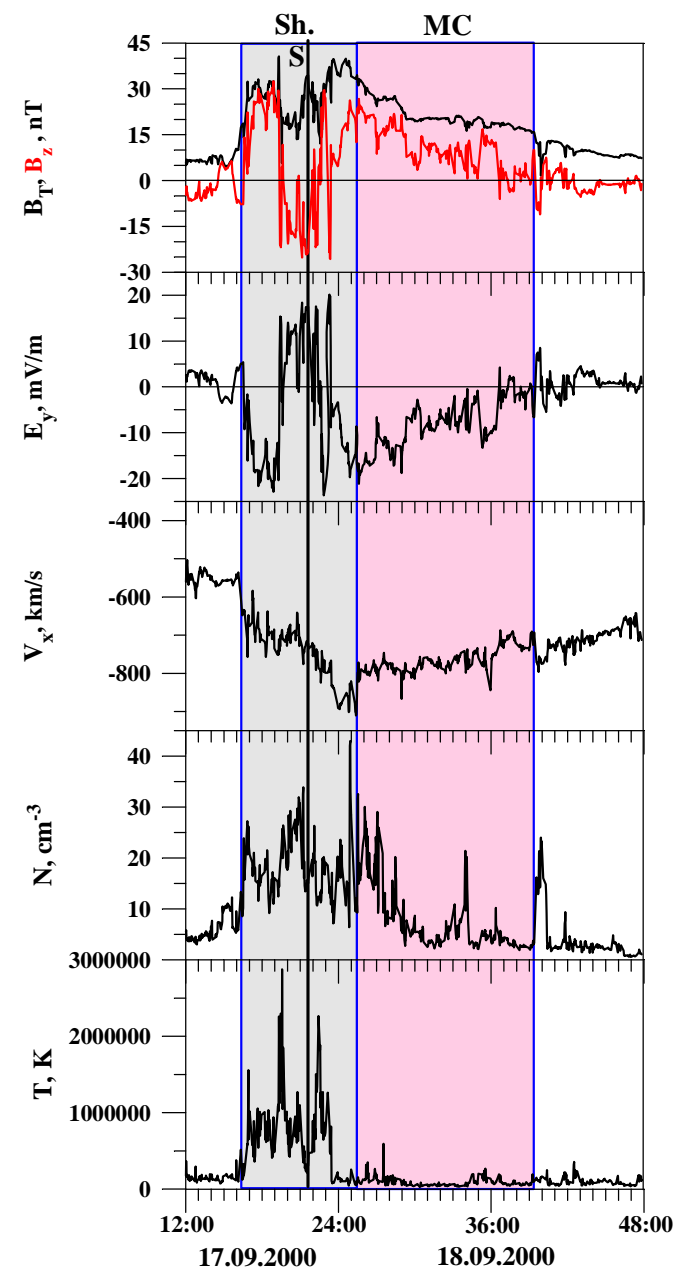

(b)

Fig. 1. Total magnetic field $B_{T}$ (black line) and north-south component $B_{Z}$ of IMF (red line), Y-component of the interplanetary electric field $E_{Y}, \mathrm{X}$-component of the solar wind velocity $V_{X}$, solar wind proton density $N$ and proton temperature $T$ for two cases of MC/Sheath observation close to $1 \mathrm{AU}$. The intervals of observation of MC and Sheath are marked by pink and grey colour, respectively. Vertical blue lines mark the start and finish of MC/Sheath structures. The times of substorm observation onboard the Polar spacecraft are marked by vertical black dashed and solid lines, where the solid line correspond to the time of maximum development of substorm, for which the Polar UVI data are shown below, in Fig. 3.

cases from 1st group the substorms have been observed no less than $2 \mathrm{~h}$ after the MC beginning. All substorms of $2 \mathrm{nd}$ group occurred in the middle or nearer to the end of Sheath region. For the examples shown in Fig. 1, the times of substorms observation by Polar during Sheath/MC structures are indicated by vertical black lines.

Recurrent streams are determined as high-speed streams, which reappear in each solar rotation, thus giving 27-days periodicity in the occurrence of these streams. The recurrent streams are characterized by increased solar wind velocity $\left(\left|V_{X}\right|>500 \mathrm{~km} / \mathrm{s}\right)$, and lower (than the average) density; the duration of these streams is $\sim 3-4$ days (e.g., Pudovkin, 1996). In front of the recurrent stream there is a region of the interaction with slower streams (CIR). CIR is determined as a region with magnetic field and plasma compression (Balogh et al., 1999).

Two examples of recurrent stream observation are shown in Fig. 2. The left (right) panels correspond to the recurrent stream passing on 11-13 January 1997 (27 February-1 March 1997). The start and finish time for the CIR and RS regions passage are determined by visual data inspection according to their properties.

We selected all substorms during the passage of recurrent streams in the period of December 1996, January and February 1997 (6 recurrent streams in total), for which Polar satellite data were available. All substorms were divided into 2 


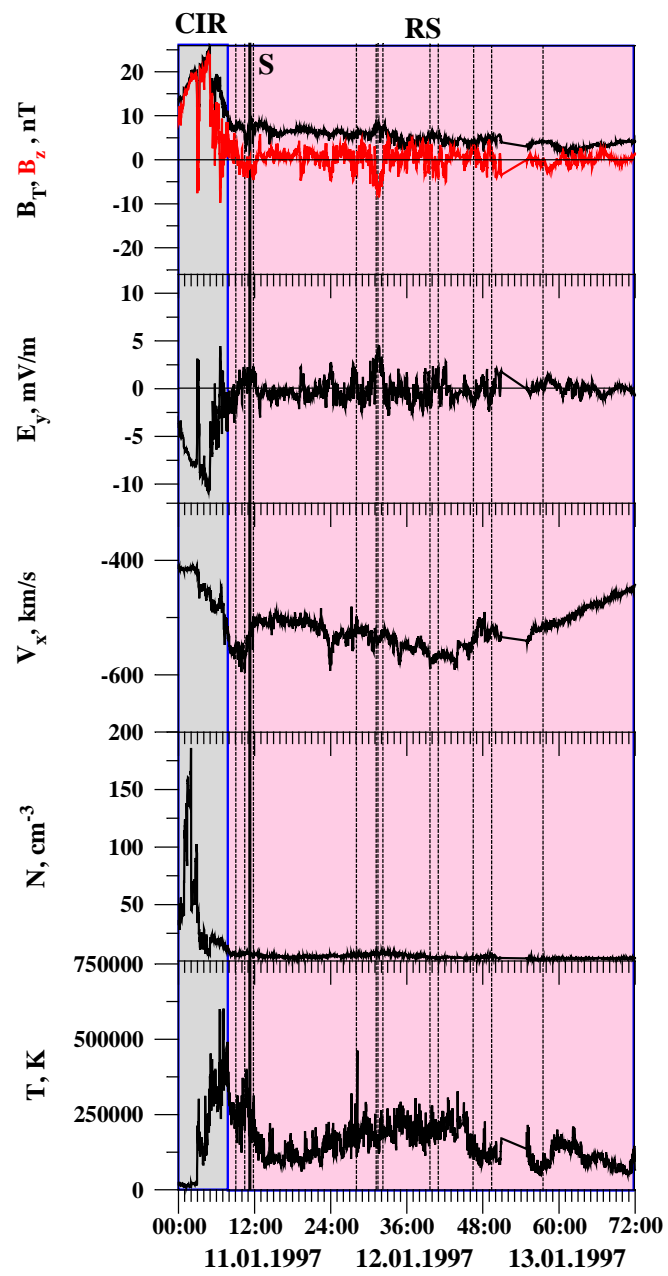

(a)

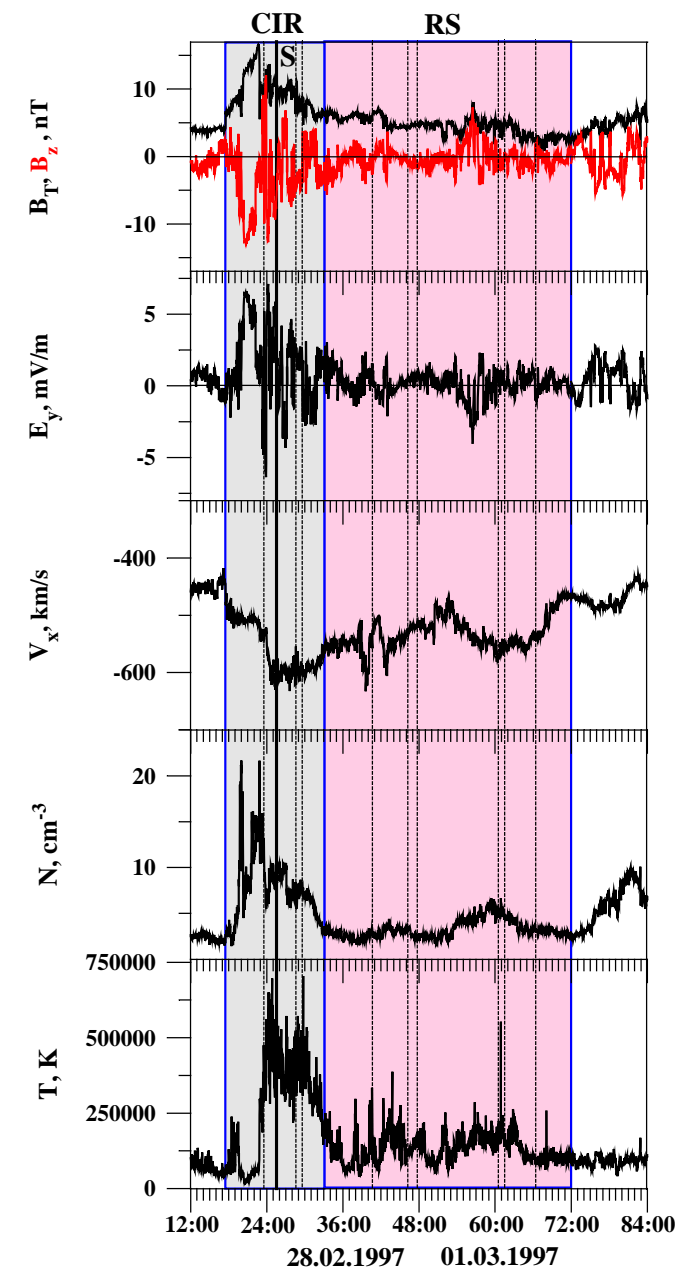

(b)

Fig. 2. The format is the same as in Fig. 1, but for the RS/SIR events. The pink and grey colours mark the intervals of observation of RS and CIR, respectively.

groups: substorms observed during the passage of the recurrent streams ( 72 events, 1 st group), and of the CIR (26 events, 2 nd group). The majority of selected substorms occur at least $2 \mathrm{~h}$ after the beginning of corresponding structure.

The total durations of the selected intervals are equal to about 440, 190, 320, and $130 \mathrm{~h}$ for the RS, CIR, MC, and Sheath structures, respectively.

\section{Results}

In Fig. 3 examples of auroral bulge development during the passage of 4 different solar wind structures, given in Figs. 12, are shown. In Fig. 3a, the substorm development during MC of 15 July 2000 (shown in Fig. 1a), is presented; in Fig. $3 b$ - the one during RS of 11 January 1997 (shown in Fig. 2a), in Fig. 3c - the one during Sheath of 17 Septem- ber 2000 (shown in Fig. 1b), and in Fig. 3d - the one during CIR of 28 February 1997 (shown in Fig. 2b). The blue curve delimits the bulge region on each auroral image. In Figs. 1-2 the vertical black solid lines indicate the time of maximum substorm development. The substorm of 15 July 2000 occurred during the main phase of the magnetic storm, under the extremely large values of the solar wind velocity, southward IMF, and dawn-dusk component of the IEF (see Fig. 1a). For this case the background level of the auroral oval luminosity before the substorm onset was equal to 158 photons $\mathrm{cm}^{-2} \mathrm{~s}^{-1}$.

In Table 1 the parameters of auroral bulge for these 4 examples are given. There are the latitudes and sizes of the bulge and the ratio between bulge sizes. We note that the bulge latitudinal size is not equal to the difference between the onset and maximum latitudes. This is because this size is measured at the moment of maximum substorm 


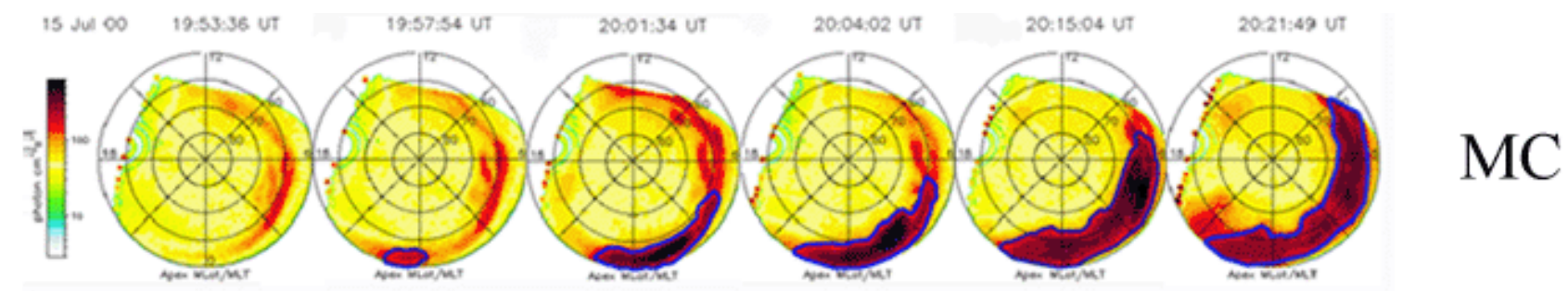

a)

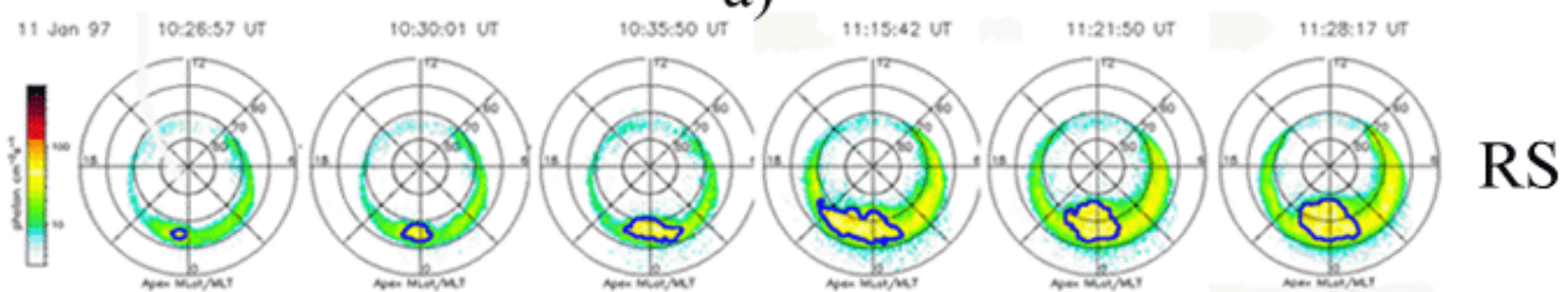

b)

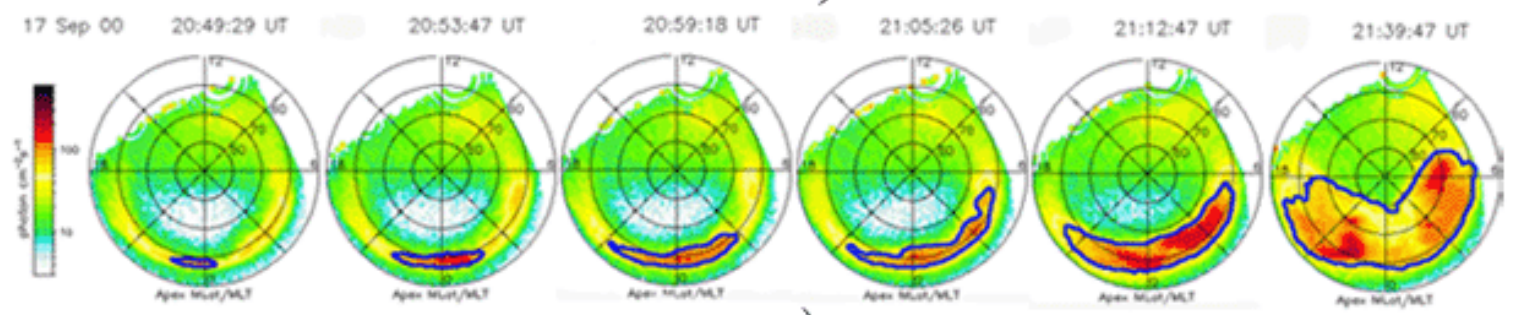

\section{Sheath}

c)

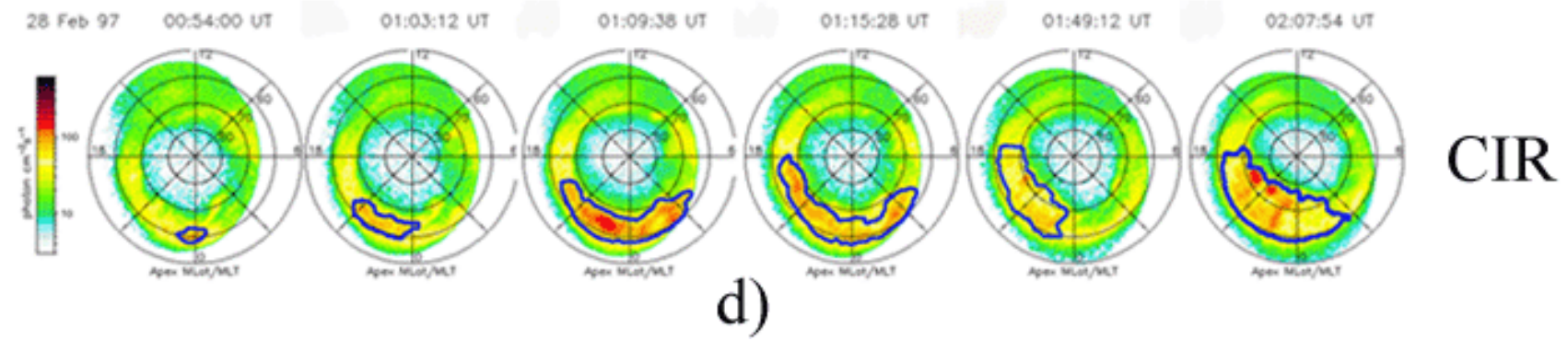

Fig. 3. Examples of substorm development by Polar UVI data during the passage of different solar wind structures: magnetic cloud (a), recurrent stream (b), Sheath (c) and CIR (d). On each auroral image the blue curve delimits the bulge region.

development. A comparative analysis of these examples shows that the auroral bulge was formed at very low geomagnetic latitudes during MC, whereas three further examples refer to the "high latitudinal" substorms, for which the poleward edge of the auroral bulge propagated up to very high latitudes $\left(\sim 80^{\circ}\right.$ CGLAT). The event shown on the upper panel of Fig. 3a is an example of substorm development under extremely high values of solar wind parameters $\left(V_{X} \sim-1022 \mathrm{~km} / \mathrm{s}, B_{Z} \sim-35 \mathrm{nT}\right)$ and the auroral bulge dynamics during magnetic storm main phase $\left(D_{s t} \sim-198\right)$. For this extreme event the ratio between longitudinal and latitudinal sizes of the bulge is maximal. It will be shown below that for the other substorms of the MC category the ratio between bulge sizes is also higher in comparison with that for substorms of the RS category. The substorm presented on Fig. $3 \mathrm{~b}$ developed under $V_{X} \sim-550 \mathrm{~km} / \mathrm{s}$ and $B_{Z} \sim-$ $1.5 \mathrm{nT}$ during a non-storm interval $\left(D_{s t} \sim-28\right)$. The substorms illustrated on the bottom panels (3c and 3d) developed under high values of solar wind parameters $\left(V_{X} \sim-\right.$ $705 \mathrm{~km} / \mathrm{s}$ and $-585 \mathrm{~km} / \mathrm{s} ; B_{Z} \sim-12.5 \mathrm{nT}$ and $\left.-5.5 \mathrm{nT}\right)$. The Sheath-associated substorm (Fig. 3c) was observed at the "initial" phase of storm $\left(D_{s t} \sim-61\right)$, whereas CIR-associated substorm (Fig. 2d) occurred during the storm main phase $\left(D_{s t} \sim-67\right)$.

As seen from Table 1, the latitudinal and longitudinal dimensions of the auroral bulge of substorms, occurred during MC, Sheath, RS, and CIR, are different. This difference manifests more clearly in Fig. 4, where these dimensions are 
Table 1. Latitudes and sizes of the auroral bulge and ratio between bulge sizes for four solar wind structures (MC, RS, Sheath and CIR).

\begin{tabular}{lccccc}
\hline $\begin{array}{l}\text { Solar wind } \\
\text { structure }\end{array}$ & $\begin{array}{c}\text { Onset latitude } \\
\left({ }^{\circ} \text { CGLAT }\right)\end{array}$ & $\begin{array}{c}\text { Maximal latitude } \\
\left({ }^{\circ} \text { CGLAT }\right)\end{array}$ & $\begin{array}{c}\text { Latitudinal size } \\
\left(L_{F}\right)\left({ }^{\circ} \text { CGLAT }\right)\end{array}$ & $\begin{array}{c}\text { Longitudinal size } \\
\left(L_{d}\right)\left({ }^{\circ} \text { CGLAT }\right)\end{array}$ & $\begin{array}{c}\text { The ratio between longitudinal and } \\
\text { latitudinal sizes }\left(L_{d} / L_{F}\right)\end{array}$ \\
\hline MC & 50.5 & 68.7 & 16.3 & 289 & 17.7 \\
RS & 64.0 & 79.3 & 18.0 & 73 & 4.1 \\
Sheath & 56.7 & 80.3 & 22.0 & 221 & 10.0 \\
CIR & 55.3 & 82.3 & 25.6 & 226 & 8.8 \\
\hline
\end{tabular}
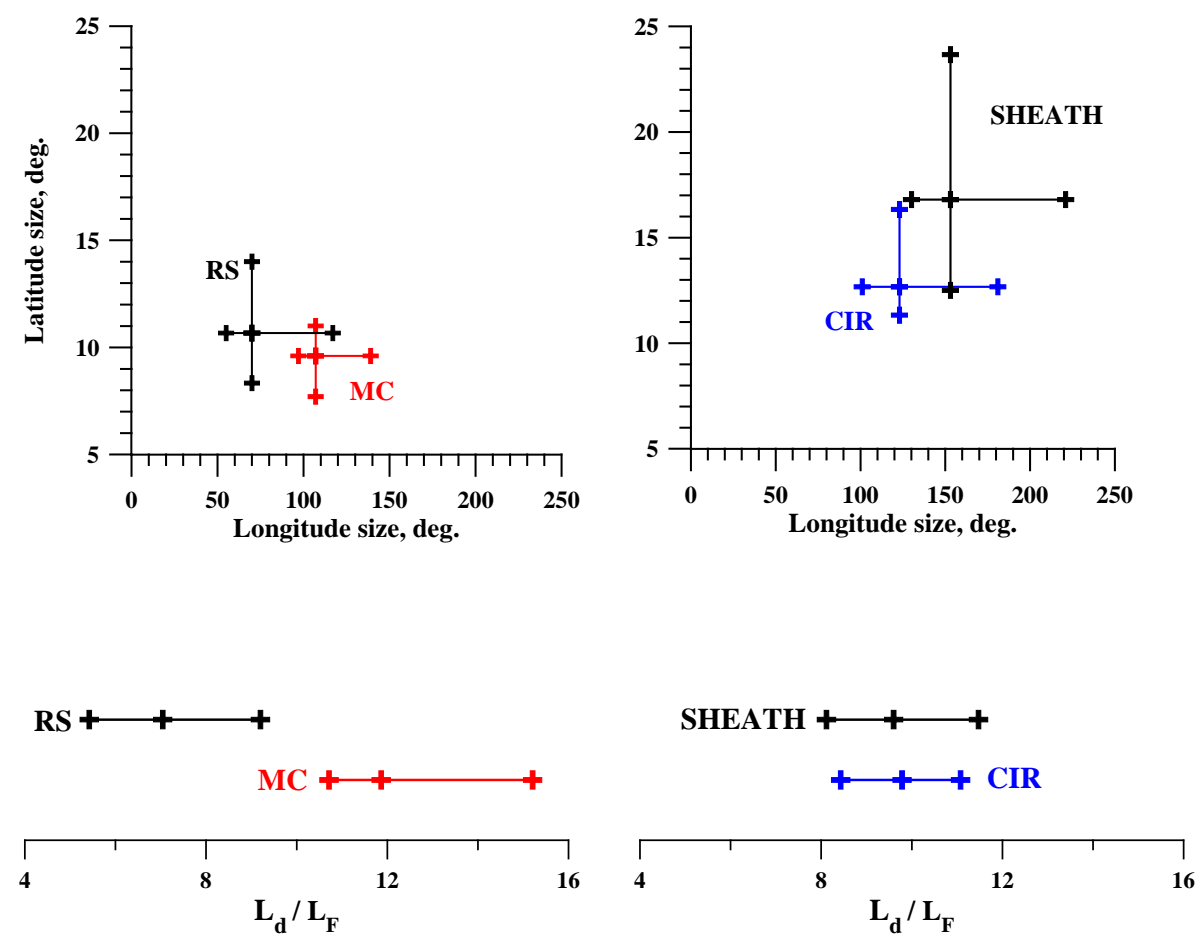

Fig. 4. The median values, first and third quartiles of distribution of auroral bulge parameters for MC and RS (left column), Sheath and CIR (right column). The upper panels show the latitudinal and longitudinal sizes, and the bottom panels present the ratio of longitudinal to latitudinal sizes.

compared. Here are presented the median values of latitudinal and longitudinal sizes, first and third quartiles of their distributions. On the left panel, one can see the longitudinal and latitudinal bulge sizes and their ratios for the substorms during MCs and RS's, on the right panel - those for CIR- and Sheath-associated substorms. For RS-associated substorms, the latitudinal size of the auroral bulge is, in average, larger than for MC-associated substorms. An opposite relationship holds true for the longitudinal sizes, the average value being higher for MC-associated substorms. The effect becomes more evident if we examine the ratio of latitudinal and longitudinal dimensions of the bulge for RS and for MC events. This ratio is rather stable and always less for MC than for RS events. In particular, this ratio for MC-associated substorm shown in Fig. 3a is 4.4 times less than that for RS-associated one given in Fig. 3b. For Sheath and CIR conditions, the sizes of the bulge are very large: the latitudinal sizes are comparable to the bulge sizes for RS-associated substorms, whereas the longitudinal sizes are comparable or larger than those for MC-associated substorms.

It is considered how the solar wind parameters influence on the latitudinal location of auroral bulge edges for MC-, CIR-, Sheath-, and RS-associated substorms. In Fig. 5 are shown the onset and maximum latitudes versus the solar wind parameters. The panels, from left to right, present the latitudes dependences on the solar wind velocity $\left|V_{X}\right|$, on the southward IMF component $B_{S}$, and on the dawn-dusk component of the interplanetary electric field. The upper (bottom) panel in Fig. 5 shows the dependencies for magnetic cloud (CIR), the second (third) panel shows the dependencies for recurrent streams (Sheath). As one can see from Fig. 5, the difference between the onset and maximum latitudes for 

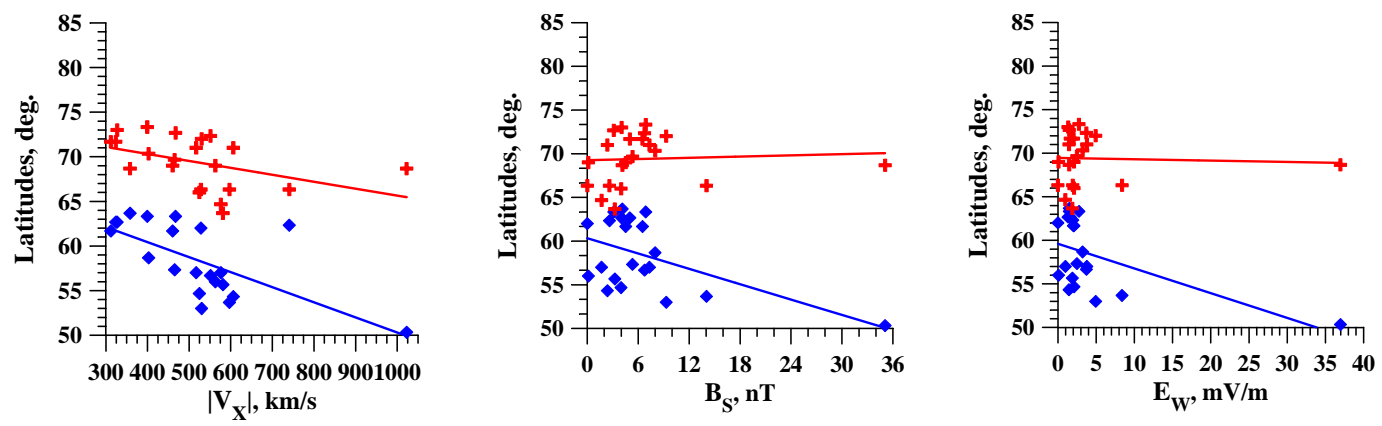

(a) MC
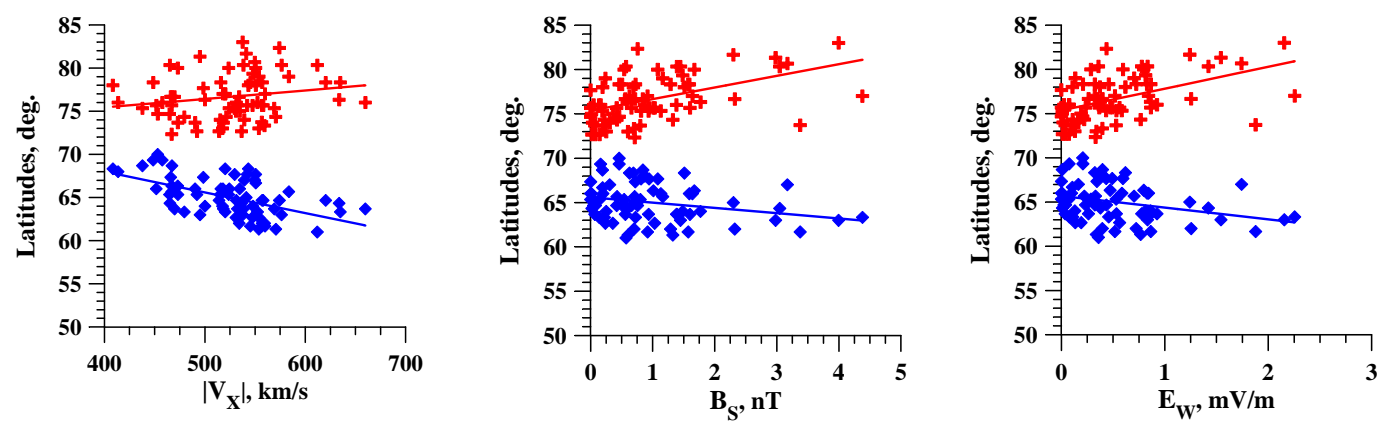

(b) RS
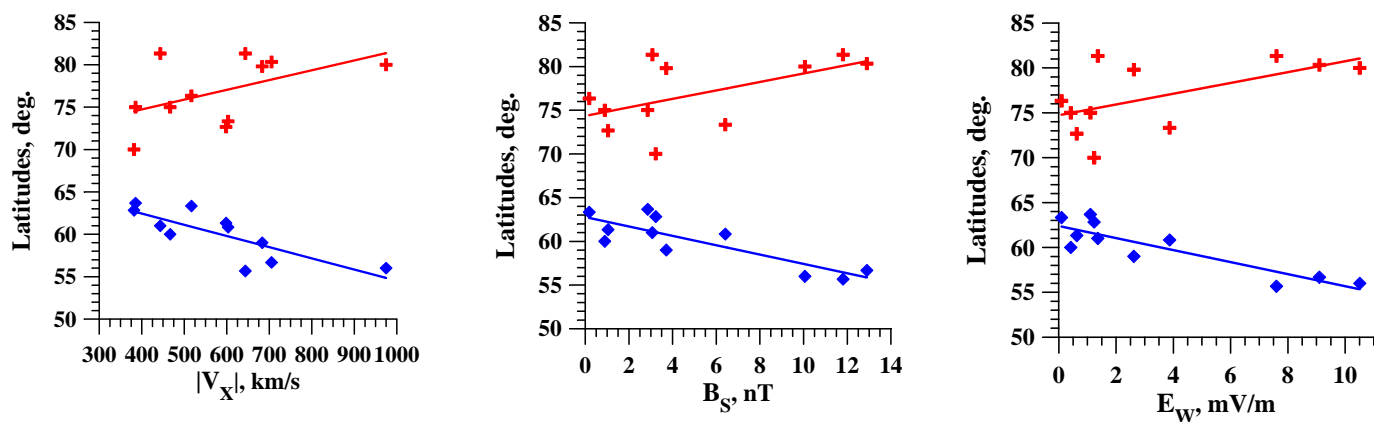

\section{(c) Sheath}
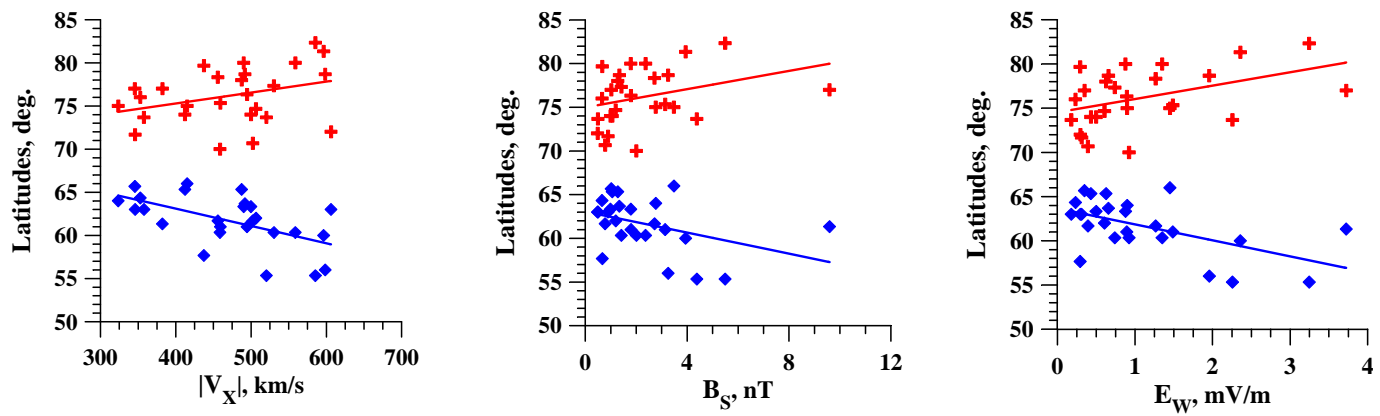

\section{(d) CIR}

Fig. 5. Onset (blue symbols and line) and maximum (red symbols and line) latitudes of auroral bulge depending on the radial component of the solar wind velocity (the left panels), the southward IMF component (the middle panels), and the dawn-dusk component of the interplanetary electric field (the right panels). The upper (bottom) panels show the dependencies for magnetic cloud (CIR), the second (third) panels show the dependencies for recurrent streams (Sheath). 
all types of substorms increases with the increase of all considered interplanetary parameters. The onset latitude decreases in all cases. For RS, CIR, and Sheath-associated substorms the maximum latitude increases with the increase of all solar wind parameters. However, for MC-associated substorms the maximum latitude decreases with the increase of the solar wind velocity $\left|V_{X}\right|$. The dependence on $B_{S}$ and $E_{W}$ is not evident because of strong impact of the outlier points with extremely large values of $B_{S}$ and $E_{W}$. However, it is possible to assert that in the MC category of substorms the maximum latitude increases slowly with the increase of $B_{S}$ and $E_{W}$ in comparison with the RS category. Indeed, exclusion of two outlier points leads to increase of the slope of the fit from 0.02 to 0.65 for dependence on $B_{S}$ and from -0.02 to 0.97 for dependence on $E_{W}$, whereas for substorms in the RS category the slope is equal to 1.31 and 2.46 for dependence on $B_{S}$ and $E_{W}$, respectively. It is also seen that the auroral bulge developed at higher latitudes during recurrent streams than during magnetic clouds.

\section{Discussion}

We found a difference in auroral bulge "geometry" for MCand RS-associated substorms. The auroral bulges of substorms that are observed during magnetic clouds are, in average, wider in longitude and narrower in latitude than those related to recurrent streams (Fig. 4). In addition, we found that during MC, the ratio of latitudinal and longitudinal dimensions of the bulge is rather stable and always less than that for RS. In other words, during MC the auroral bulge has a relatively small latitudinal size, for it is more extended along longitude.

The reason for such different "geometry" of the auroral bulge may be in different configuration of the geomagnetic tail during the MC and RS. As a rule, the magnetic clouds, as well as the Sheath and CIR, are associated with geomagnetic storms (e.g., Wilson, 1987; Tsurutani et al., 2006), while during the passage of recurrent streams, the storms do not typically occur. This is the case for the events examined in this study: all MC-associated substorms here considered were observed against a geomagnetic storm background, at either storm main phase or recovery phase $\left(D_{s t} \sim-110\right)$; the substorms that occurred during Sheath, referred to the beginning of storm $\left(D_{s t} \sim-40\right)$; the substorms observed during recurrent streams corresponded to non-storm conditions $\left(D_{s t} \sim-18\right)$; the CIR-associated substorms referred to either non-storm intervals $\left(D_{s t} \sim-20\right)$ or to CIR-associated storm development $\left(D_{s t} \sim-67\right)$.

The configuration of the geomagnetic tail strongly changes under storm conditions. As shown by Pulkkinen et al. (2006), the storm-time geomagnetic tail is more stretched. Pulkkinen et al. (2006) considered the substorms occurred during the passage of MC on 22 October 2001 and showed that under $\mathrm{MC}$-associated storms, an intense thin current sheet forms that occupies a wider MLT sector of the near Earth tail. The formation of an intense thin current sheet provides favourable conditions for driving magnetic reconnection in this region, which may be a cause of substorm (e.g., Yahnin et al., 2006). Therefore, the substorm can develop in a wider longitudinal sector, without propagating far down the magnetospheric tail.

Different "geometry" of auroral bulge under MC and RS conditions, can explain the fact that substorms at high latitudes predominantly occur during the period of solar minimum (Dmitrieva and Sergeev, 1984; Despirak et al., 2008). It is known that substorm expansion to high latitudes is associated with large values of the solar wind velocity and of the southward IMF $B_{S}$ component (Gussenhoven, 1982; Dmitrieva and Sergeev, 1984; Weatherwax et al., 1997; Zverev et al., 1979). However, during magnetic clouds even if the values of $\left|V_{X}\right|$ and $B_{S}$ are extremely high, the auroral bulge develops at low latitudes. The bulge area is a measure of magnetic flux at the ionospheric level, the flux through the bulge being equal to that stored in the tail before the substorm onset (Shukhtina et al., 2005). On the other hand, this stored flux is proportional to the solar wind electric field, driving reconnection on the magnetopause. Yahnin et al. (2004) showed that for the fixed values of the solar wind electric field and of IMF $B_{S}$, the auroral bulge area is nearly the same in the periods of solar minimum and solar maximum. Still, as one can see, the auroral bulge "geometry" is different. During MCs, the substorm can develop in a wider longitudinal sector, and then larger bulge area (associated with large reconnected magnetic flux under high values of $\left|V_{X}\right|$ and $B_{S}$ ) does not necessarily mean a substorm expansion to very high latitudes. During RS's, the auroral bulge "geometry" is different: the bulge is more extended in latitude and narrower in longitude; the ratio of the longitudinal and latitudinal sizes several times decreasing. Thus the RS-associated bulge of the same area will develop at higher latitudes. In addition, as shown in this study, the substorms during MCs start at lower latitudes than those during RS's (Fig. 5, see also Yahnin et al., 2004; Gérard et al., 2004). Under solar minimum (maximum) conditions, it is RS's (MCs) that majorly contribute to geomagnetic activity (e.g., Richardson et al., 2000). Accordingly, substorms at high latitudes predominantly occur in the periods of solar minimum. They are very rare in the periods of solar maximum.

It should be noted that the "high-latitude" substorms can also occur during Sheath events. However, they do not contribute much to the overall picture of high-latitude substorm occurrence under solar maximum conditions, since the duration of such events is short compared to that of magnetic cloud events (Despirak et al., 2008).

The area of the auroral bulges associated with Sheath and CIR is the largest. Such bulges are most extended in both latitude and longitude. Perhaps, this is due to the influence of the compressed, highly dense solar wind plasma (Yermolaev et al., 2005). The auroral bulge area can considered as a measure of the magnetic flux dissipated in the magnetotail during 
substorm and also a measure of energy dissipation both in the magnetotail and ionosphere (Yahnin et al., 2006). Hence one can expect that larger dissipated flux and also larger energy dissipation in the magnetotail must occur during substorms associated with Sheath and CIR than during substorms in the course of $\mathrm{MC}$ when the input energy and driving are largest. This result is in accordance with the conclusion of the recent paper by Palmroth et al. (2007). In this paper, the solar wind - magnetosphere coupling efficiency as response to the solar wind dynamic pressure impulses was investigated. It was shown, that the magnetosphere uses the energy of a weaker driver more efficiently, whereas during the stronger drivers the energy is more inefficiently used. However, the consideration of this effect is beyond the scope of the present paper and requires further investigations.

\section{Conclusion}

The auroral bulge "geometry" differs for substorms occurring during the solar wind recurrent streams and magnetic clouds. This is evidenced by difference in the ratio of auroral bulge longitudinal and latitudinal dimensions. In particular, during $\mathrm{MC}$ conditions the auroral bulge is confined in latitude and extended in longitude. We relate this to storm-like configuration of the near-Earth magnetotail, which is very stretched in wide range of MLT.

Acknowledgements. Polar and WIND data used in this study were taken from the official Internet sites of UVI, SWE, MFI (http:// cdaweb.gsfc.nasa.gov/cdaweb/istp_public/; http://uvisun.msfc.nasa. gov/UVI/brwseimages.html). We are grateful to G. Parks, K. Ogilvie, and R. Lepping, the heads of the experiments conducted with these instruments. The work was supported by the Presidium of the Russian Academy of Sciences (RAS) through the basic research program "Solar activity and physical processes in the SunEarth system" and by the Division of Physical Sciences of RAS through the program "Plasma processes in the solar system". The study is part of a joint Russian-Bulgaria project "The influence of solar activity and solar wind streams on the magnetospheric disturbances, particle precipitations and auroral emissions" of PGI RAS and STIL-BAS under the program for fundamental space research between RAS and BAS.

Topical Editor R. Nakamura thanks M. M. E. Palmroth and another anonymous referee for their help in evaluating this paper.

\section{References}

Akasofu, S.-I.: The development of the auroral substorm, Planet. Space Sci., 12, 273-282, 1964.

Balogh, A., Gosling, J. T., Jokipii, J. R., Kallenbach, R., and Kunow, H.: Corotating interaction regions. Proceedings. ISSI Workshop, Bern (Switzerland), 6-13 June 1998, Space Sci. Rev., 89(1-2), XIV + 411, 1999.

Burlaga, L. F., Klein, L., Sheeley Jr., N. R., Michels, D. J., Howard, R. A., Koomen, M. J., Schwenn, R., and Rosenbauer, H.: A mag- netic cloud and a coronal mass ejection, Geophys. Res. Lett., 9, 1317-1320, 1982.

Despirak, I. V., Lubchich, A. A., Biernat, H. K., and Yahnin, A. G.: Poleward expansion of the westward electrojet depending on the solar wind and IMF parameters, Geomagnetism and Aeronomy, 48, 284-292, 2008.

Dmitrieva, N. P. and Sergeev, V. A.: The appearance of an auroral electrojet at polar cap latitudes: The characteristics of the phenomenon and the possibility of its use in predicting large-scale high-speed solar wind streams, Magnitosfernye Issledovaniia, 3, 58-66, 1984 (in Russian).

Gérard, J.-C., Hubert, B., Grard, A., Meurant, M., and Mende, S. B.: Solar wind control of auroral substorm onset locations observed with the IMAGE-FUV imagers, J. Geophys. Res., 109, A03208, doi:10.1029/2003JA010129, 2004.

Gonzalez, W. D., Gonzalez, A. L. C., and Tsurutani, B. T.: Dualpeek solar cycle distribution of intense geomagnetic storms, Planet. Space Sci., 38(2), 181-187, 1990.

Gussenhoven, M. S.: Extremely high latitude auroras, J. Geophys. Res., 87, 2401-2412, 1982.

Huttunen, K. E. J., Koskinen, H. E. J., Karinen, A., and Mursula, K.: Asymmetric development of magnetospheric storms during magnetic clouds and sheath regions, Geophys. Res. Lett., 33, L06107, doi:10.1029/2005GL024894, 2006.

Krieger, A. S., Timothy, A. F., and Roelof, E. C.: A coronal hole and its identification as the source of a high velocity solar wind stream, Solar Phys., 29(2), 505-525, 1973.

McPherron, R. L.: Growth phase of magnetospheric substorms, J. Geophys. Res., 75, 5592-5599, 1970.

Miyashita, Y., Machida, S., Liou, K., Mukai, T., Saito, Y., Meng, C.I., and Parks, G. K.: Relationship between magnetotail variations and auroral activities during substorms, J. Geophys. Res., 108, 1022, doi:10.1029/2001JA009175, 2003.

Palmroth, M., Partamies, N., Polvi, J., Pulkkinen, T. I., McComas, D. J., Barnes, R. J., Stauning, P., Smith, C. W., Singer, H. J., and Vainio, R.: Solar wind-magnetosphere coupling efficiency for solar wind pressure impulses, Geophys. Res. Lett., 34, L11101, doi:10.1029/2006GL029059, 2007.

Pudovkin, M. I.: Solar wind, Soros Educational Journal, 12, 87-94, 1996 (in Russian).

Pulkkinen, T. I., Ganushkina, N. Y., Tanskanen, E. I., Kubyshkina, M., Reeves, G. D., Thomsen, M. F., Russel, C. T., Singer, H. J., Slavin, J. A., and Gjerloev, J.: Magnetospheric current systems during stormtime sawtooth events, J. Geophys. Res., 111, A11S17, doi:10.1029/2006JA011627, 2006.

Pulkkinen, T. I., Partamies, N., Huttunen, K. E. J., Reeves, G. D., and Koskinen, H. E. J.: Differences in geomagnetic storms driven by magnetic clouds and ICME sheath regions, Geophys. Res. Lett., 34, L02105, doi:10.1029/2006GL027775, 2007.

Richardson, I. G., Cliver, E. W., and Cane, H. V.: Sources of geomagnetic activity over the solar cycle: Relative importance of coronal mass ejections, high-speed streams, and slow solar wind, J. Geophys. Res., 105, 18203-18213, 2000.

Sergeev, V. A., Yakhnin, A. G., and Dmitrieva, N. P.: Substorm in the polar cap - the effect of high-velocity streams of the solar wind, Geomagnetism and Aeronomy, 19, 1121-1122. 1979 (in Russian).

Shukhtina, M. A., Dmitrieva, N. P., Popova, N. G., Sergeev, V. A., Yahnin, A. G., and Despirak, I. V.: Observational evidence of 
the loading-unloading substorm scheme, Geophys. Res. Lett., 32, LI7107, doi:10.1029/2005GL023779, 2005.

Tsurutani, B. T. and Gonzalez, W. D.: A new perspective on the relationship between substorms and magnetic storms, Adv. Geosci., V 8: Solar Terrestrial (2006), edited by: Ip, W.-H. and Duldig, M., published by World Scientific Co., Pte. Ltd., Singapore, p. 25-45, 2006.

Tsurutani, B. T., Gonzalez, W. D., Gonzalez, A. L. C., Guarnieri, F. L., Gopalswamy, N., Grande, M., Kamide, Y., Kasahara, Y., Lu, G., Mann, I., McPherron, R., Soraas, F., and Vasyliunas, V.: Corotating solar wind streams and recurrent geomagnetic activity: A review, J. Geophys. Res., 111, A07S01, doi:10.1029/2005JA011273, 2006.

Wang, Y.-M. and Sheeley Jr., N. R.: Global evolution of interplanetary sector structure, coronal holes, and solar wind streams during 1976-1993: Stackplot displays based on solar magnetic observations, J. Geophys. Res., 99, 6597-6608, 1994.

Weatherwax, A. T., Rosenberg, T. J., Maclennan, C. G., and Doolittle, J. H.: Substorm precipitation in the polar cap and associated Pc 5 modulation, Geophys. Res. Lett., 24, 579-582, 1997.

Webb, D. F. and Howard, R. A.: The solar cycle variation of coronal mass ejections and the solar wind mass flux, J. Geophys. Res., 99, 4201-4220, 1994.
Wilson, R. M.: Geomagnetic response to magnetic clouds, Planet. Space Sci., 35, 329-335, 1987.

Yahnin, A. G., Despirak, I. V., Lyubchich, A. A., and Kozelov, B. V.: Solar wind control of the auroral bulge expansion, Proceedings of the 7th International Conference on Substorms, 31-34, Helsinki, 2004.

Yahnin, A. G., Despirak, I. V., Lubchich, A. A., Kozelov, B. V., Dmitrieva, N. P., Shukhtina, M. A., and Biernat, H. K.: Relationship between substorm auroras and processes in the near-earth magnetotail, Space Sci. Rev., 122, 97-106, 2006.

Yermolaev, Yu. I., Yermolaev, M. Yu., Zastenker, G. N., Zelenyi, L. M., Petrukovich, A. A., and Sauvand, J.-A.: Statistical studies of geomagnetic storm dependencies on solar and interplanetary events: a review, Planet. Space Sci., 53, 189-196, 2005.

Yermolaev, Yu. I. and Yermolaev, M. Yu.: Statistic study on the geomagnetic storm effectiveness of solar and interplanetary events, Adv. Space Res., 37, 1175-1181, 2006.

Zverev, V. L., Starkov, G. V., and Feldstein, Y. I.: Influences of the interplanetary magnetic field on the auroral dynamics, Planet. Space Sci., 27, 665-667, 1979. 